\title{
Experimental test of quantum contextuality in neutron interferometry
}

\author{
H. Bartosik ${ }^{1}$, J. Klepp ${ }^{1}$, C. Schmitzer ${ }^{1}$, S. Sponar ${ }^{1}$, A. Cabello ${ }^{2}$, H. Rauch ${ }^{1,3}$, and Y. Hasegawa ${ }^{1}$ \\ ${ }^{1}$ Atominstitut der Österreichischen Universitäten, Stadionallee 2, A-1020 Wien, Austria \\ ${ }^{2}$ Departamento de Física Aplicada II, Universidad de Sevilla, E-41012 Sevilla, Spain \\ ${ }^{3}$ Institut Laue-Langevin, Boite Postale 156, F-38042 Grenoble Cedex 9, France
}

(Dated: August 7, 2018)

\begin{abstract}
We performed an experimental test of the Kochen-Specker theorem based on an inequality derived from the Peres-Mermin proof, using spin-path (momentum) entanglement in a single neutron system. Following the strategy proposed by Cabello et al. [Phys. Rev. Lett. 100, 130404 (2008)], a Belllike state was generated and three expectation values were determined. The observed violation $2.291 \pm 0.008 \not \leq 1$ clearly shows that quantum mechanical predictions cannot be reproduced by noncontextual hidden variables theories.
\end{abstract}

PACS numbers: 03.65.Ud, 03.75.Dg, 07.60.Ly, 42.50.Xa

There are two powerful arguments against the possibility of extending quantum mechanics (QM) into a more fundamental theory yielding a deterministic description of nature. One is the experimental violation of Bell inequalities [1, 2, , 3, , 4, [5, [6], which discards local hidden-variable theories as a possible extension to QM. The other is the Kochen-Specker (KS) theorem 7], which stresses the incompatibility of QM with a larger class of hidden-variable theories, known as noncontextual hidden-variable theories (NCHVTs). By definition, NCHVTs assume that the result of a measurement of an observable is predetermined and independent of a suitable (previous or simultaneous) measurement of any other compatible (i.e., comeasurable) observable. While the original proof of the KS theorem is rather complicated, simplified versions have been proposed by Peres [8] and Mermin [9, 10]. These proofs can be converted into experimentally testable inequalities, valid for any NCHVT, but violated by QM [11, 12].

Since the first observation of neutron self-interference [13], neutron optical experiments have been serving as an established method for investigating the foundations of quantum mechanics. In particular, neutron interferometry allows the observation of quantum mechanical phenomena on a macroscopic scale [14]. Studies on entanglement between 2 degrees of freedom of single neutrons confirmed the violation of Bell-like inequalities [6]. A complete tomographic reconstruction of density matrices was performed [15]. Recent developments on the coherent manipulation of the energy degree of freedom of single neutrons [16] provide the basis for the generation of triply entangled quantum states, where the peculiarity of a Greenberger-Horne-Zeilinger-like state was exhibited [17]. In addition to the interferometric scheme, the nonadditivity of the mixed state phase was demonstrated in neutron polarimetry [18]. Neutrons in the ultralowenergy regime, i.e., ultracold neutrons (UCNs), can be stored for several minutes, which allows for novel explorations: the stability of the Berry phase was studied by tuning the evolution time during the storage [19]. At a stage of experimental tests of quantum contextuality, the spin-path (momentum) entanglement in single neutrons allowed for demonstrating Kochen-Specker-like phenomena [20]. Further theoretical analysis revealed a more advanced scheme and an experiment with neutron interferometry was proposed [11. In this Letter we report on an improved experimental test of the KS theorem using single neutrons entangled in 2 degrees of freedom.

For the proof of the Kochen-Specker theorem, we consider single neutrons prepared in a maximally entangled Bell-like state described by the wave function

$$
|\Psi\rangle=\frac{1}{\sqrt{2}}(|\downarrow\rangle \otimes|I\rangle-|\uparrow\rangle \otimes|I I\rangle),
$$

where $|\uparrow\rangle$ and $|\downarrow\rangle$ denote the up-spin and down-spin eigenstates, and $|I\rangle$ and $|I I\rangle$ the two beam paths in a neutron interferometer. We define Pauli-type operators for the spin and path degree of freedom, e.g., $\sigma_{x}^{s}=|\uparrow\rangle\langle\downarrow|+| \downarrow\rangle\langle\uparrow|$ and $\sigma_{x}^{p}=|I\rangle\langle I I|+| I I\rangle\langle I|$, where $s$ stands for spin and $p$ for path. The proof is based on the six observables $\sigma_{x}^{s}, \sigma_{x}^{p}, \sigma_{y}^{s}, \sigma_{y}^{p}, \sigma_{x}^{s} \sigma_{y}^{p}$, and $\sigma_{y}^{s} \sigma_{x}^{p}$, and the following five quantum mechanical predictions for the state $|\Psi\rangle$,

$$
\begin{aligned}
\sigma_{x}^{s} \cdot \sigma_{x}^{p}|\Psi\rangle & =-|\Psi\rangle, \\
\sigma_{y}^{s} \cdot \sigma_{y}^{p}|\Psi\rangle & =-|\Psi\rangle, \\
\sigma_{x}^{s} \sigma_{y}^{p} \cdot \sigma_{x}^{s} \cdot \sigma_{y}^{p}|\Psi\rangle & =+|\Psi\rangle, \\
\sigma_{y}^{s} \sigma_{x}^{p} \cdot \sigma_{y}^{s} \cdot \sigma_{x}^{p}|\Psi\rangle & =+|\Psi\rangle, \\
\sigma_{x}^{s} \sigma_{y}^{p} \cdot \sigma_{y}^{s} \sigma_{x}^{p}|\Psi\rangle & =-|\Psi\rangle .
\end{aligned}
$$

Reproducing these predictions in the framework of NCHVTs requires assigning predetermined measurement results to each of the six observables [note that we introduced $(\cdot)$ to separate operators which, in NCHVTs, correspond to observables with predetermined measurement results]. The inconsistency arising in any attempt to ascribe the predefined values -1 or +1 to each and every of the six observables can be easily seen by multiplying Eqs. (2a)-(2e). Since each observable appears twice, the left hand sides give +1 while the product of the right hand sides is -1 .

An ideal experiment for verifying this contradiction would be to confirm each of the five predictions of QM, Eqs. (2a)-(2e). However, it is not possible to obtain perfect correlations in a real experiment. An experimentally 
testable inequality can be derived from the linear combination of the five expectation values with the respective quantum mechanical predictions as linear coefficients. It can be shown that in any NCHVT

$$
\begin{aligned}
-\left\langle\sigma_{x}^{s} \cdot \sigma_{x}^{p}\right\rangle & -\left\langle\sigma_{y}^{s} \cdot \sigma_{y}^{p}\right\rangle+\left\langle\sigma_{x}^{s} \sigma_{y}^{p} \cdot \sigma_{x}^{s} \cdot \sigma_{y}^{p}\right\rangle \\
& +\left\langle\sigma_{y}^{s} \sigma_{x}^{p} \cdot \sigma_{y}^{s} \cdot \sigma_{x}^{p}\right\rangle-\left\langle\sigma_{x}^{s} \sigma_{y}^{p} \cdot \sigma_{y}^{s} \sigma_{x}^{p}\right\rangle \leq 3,
\end{aligned}
$$

while the prediction of QM is 5. In order to test this inequality one needs to perform five experiments according to the five different experimental contexts represented by Eqs. (2a)-2ed . It is important to note here that (since we would like to test quantum contextuality) the six measurement apparatuses used for measuring the six observables must be the same irrespective of the experimental context in which they appear.

As already pointed out in the previous Letter [11], the five experiments of Eqs. (2a)-(2e) contribute in different ways to the proof. While Eqs. (2a), (2b), and (2e) represent state-dependent predictions relying on the specific properties of the state $|\Psi\rangle$, Eqs. (2C) and (2d) are stateindependent predictions which hold in any NCHVT. In other words, in any NCHVT, $\left\langle\sigma_{x}^{s} \sigma_{y}^{p} \cdot \sigma_{x}^{s} \cdot \sigma_{y}^{p}\right\rangle=1$ and $\left\langle\sigma_{y}^{s} \sigma_{x}^{p} \cdot \sigma_{y}^{s} \cdot \sigma_{x}^{p}\right\rangle=1$. Therefore, any NCHVT must satisfy not only inequality (3), but also the following inequality:

$$
-\left\langle\sigma_{x}^{s} \cdot \sigma_{x}^{p}\right\rangle-\left\langle\sigma_{y}^{s} \cdot \sigma_{y}^{p}\right\rangle-\left\langle\sigma_{x}^{s} \sigma_{y}^{p} \cdot \sigma_{y}^{s} \sigma_{x}^{p}\right\rangle \leq 1 .
$$

A violation of inequality (4) reveals quantum contextuality as long as the measurements of the six observables involved in (4) are performed in such a way that it would be possible to determine also $\left\langle\sigma_{x}^{s} \sigma_{y}^{p} \cdot \sigma_{x}^{s} \cdot \sigma_{y}^{p}\right\rangle$ and $\left\langle\sigma_{y}^{s} \sigma_{x}^{p} \cdot \sigma_{y}^{s} \cdot \sigma_{x}^{p}\right\rangle$, at least in principle. In this Letter, we report on an experimental test of inequality (44), including a prescription of how the measurement apparatuses used in our experiments can be combined for realizing a test of inequality (3).

The experiment was carried out at the perfect-crystal neutron optics beamline S18 at the high flux reactor of the Institute Laue-Langevin (ILL). A triple Laue interferometer setup (see Fig. 1) similar to previous neutron interferometric experiments [16] was used. By means of a Si perfect-crystal monochromator, a neutron beam with a mean wavelength of $\lambda_{0}=1.92 \AA\left(\Delta \lambda / \lambda_{0} \sim 0.02\right)$ is selected. The incident beam is confined to a beam cross section of $5 \times 5 \mathrm{~mm}^{2}$ and polarized in the vertical direction using the spin dependent birefringence in two sequential magnetic prisms. Because of the angular separation of the two sub-beams, only up-spin neutrons $(|\uparrow\rangle)$ meet the Bragg condition at the first interferometer plate and are split coherently into the two spatially separated paths, $|I\rangle$ and $|I I\rangle$. Together with a radio-frequency (RF) spin flipper in path $|I\rangle$, denoted as $\mathrm{RF}_{\omega}^{I}$, the first half of the interferometer is used for the generation of the maximally entangled Bell-like state $|\Psi\rangle$ [Eq. (1)]. A parallel-sided Si plate serves as a phase shifter for the path degree of freedom prior to the coherent recombination of the two paths at the third interferometer plate. Only neutrons emerging the interferometer in the forward direction ( $\mathrm{O}$ beam)

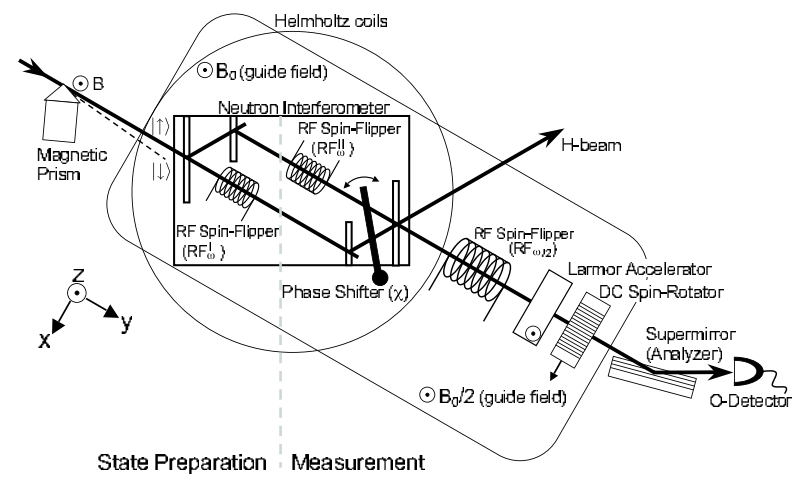

FIG. 1: Experimental setup: The maximally entangled Bell state $|\Psi\rangle$ is generated in the first half of a skew-symmetric interferometer. The second half of the interferometer together with a phase shifter serves as a path measurement apparatus. A spin analysis system in the $\mathrm{O}$ beam allows for the selection of neutrons with certain spin properties. The spin flipper in path $|I I\rangle$ is required for the measurement of the product observable $\left\langle\sigma_{x}^{s} \sigma_{y}^{p} \cdot \sigma_{y}^{s} \sigma_{x}^{p}\right\rangle$.

are used for the measurements. As explained below, our experiment requires a second $\mathrm{RF}$ flipper in the interferometer $\left(\mathrm{RF}_{\omega}^{I I}\right)$ and another $\mathrm{RF}$ flipper in the $\mathrm{O}$ beam operated at half frequency $\left(\mathrm{RF}_{\omega / 2}\right)$. Two pairs of watercooled Helmholtz coils create a fairly uniform magnetic guide field $B_{0} \hat{z}$ of $B_{0} \simeq 20 \mathrm{G}$ and $B_{0} / 2 \simeq 10 \mathrm{G}$ in the region of the interferometer and alongside the $\mathrm{O}$ beam, respectively. A spin analyzing supermirror (transmitting up-spin neutrons only) in combination with additional direct current (DC) spin rotators enable arbitrary measurements of the spin degree of freedom in the $\mathrm{O}$ beam: neutrons with the selected spin properties are counted in the subsequent $\mathrm{O}$ detector (efficiency $>99 \%$ ).

The first term in inequality (4) requires the measurement of $\sigma_{x}^{s}$ together with $\sigma_{x}^{p}$. Here, $\mathrm{RF}_{\omega / 2}$ in the $\mathrm{O}$ beam is needed for compensating the energy difference due to the spin flip at $\mathrm{RF}_{\omega}^{I}$ [16], while the second $\mathrm{RF}$ flipper in the interferometer, $\mathrm{RF}_{\omega}^{I I}$, is turned off. For measuring the path observable, i.e., $\sigma_{x}^{p}$, the phase shifter is adjusted to induce a relative phase $(\chi)$ between the two paths $|I\rangle$ and $|I I\rangle$. Settings of $\chi=0$ and $\chi=\pi$ in the path state $|\Psi(\chi)\rangle_{p}=\frac{1}{\sqrt{2}}\left(|I\rangle+e^{i \chi}|I I\rangle\right)$ correspond to the projections to $|+x\rangle_{p}$ and $|-x\rangle_{p}$, the two eigenstates of $\sigma_{x}^{p}$, respectively. The spin analysis in the $x-y$ plane is accomplished by the combination of the Larmor accelerator DC coil inducing a Larmor phase $\alpha$, the DC spin rotator tuned to a $\pi / 2$ rotation and the analyzing supermirror. This configuration allows for the selection of neutrons in the spin state $|\Psi(\alpha)\rangle_{s}=\frac{1}{\sqrt{2}}\left(|\uparrow\rangle+e^{i \alpha}|\downarrow\rangle\right)$. Spin analysis in arbitrary directions of the $x$ - $y$ plane can be realized by adequately adjusting the Larmor phase $\alpha$ between 0 and $2 \pi$. For example, the projections to $|+x\rangle_{s}$ and $|-x\rangle_{s}$, the two eigenstates of $\sigma_{x}^{s}$, correspond to $\alpha=0$ and $\alpha=\pi$, respectively. The experimental setup for the second term in inequality (4) is identical with the one for the first term, but the measurement of $\sigma_{y}^{s}$ together with 
$\sigma_{y}^{p}$ is achieved with settings of $\chi=\frac{\pi}{2}, \frac{3 \pi}{2}$ and $\alpha=\frac{\pi}{2}, \frac{3 \pi}{2}$. Typical intensity oscillations for the successive measurement of the path and the spin component are shown in Fig. 2. Clear sinusoidal dependence of the intensity on the relative phase shift $\chi$ is observed. The corresponding expectation values are then derived from the relation

$$
E(\alpha, \chi)=\frac{N(\alpha, \chi)+N(\alpha+\pi, \chi+\pi)-N(\alpha+\pi, \chi)-N(\alpha, \chi+\pi)}{N(\alpha, \chi)+N(\alpha+\pi, \chi+\pi)+N(\alpha+\pi, \chi)+N(\alpha, \chi+\pi)},
$$

where $N(\alpha, \chi)$ denotes the neutron count rate at the joint projection to the spin state $|\Psi(\alpha)\rangle_{s}$ and the path state $|\Psi(\chi)\rangle_{p}$. The required count rates at appropriate settings of $\alpha$ and $\chi$ (indicated by the vertical dashed lines in Fig. 2) are determined from least squares fits. Each measurement was carried out 3 times in order to reduce statistical errors. All errors of the fit parameters and the experimentally unavoidable phase drifts are included in the error estimation. In this way, we obtain the expectation values $\left\langle\sigma_{x}^{s} \cdot \sigma_{x}^{p}\right\rangle \equiv E(0,0)=-0.679 \pm 0.005$ and $\left\langle\sigma_{y}^{s} \cdot \sigma_{y}^{p}\right\rangle \equiv E\left(\frac{\pi}{2}, \frac{\pi}{2}\right)=-0.682 \pm 0.005$.

The third term in inequality (4) requires the measurement of $\sigma_{x}^{s} \sigma_{y}^{p}$ together with $\sigma_{y}^{s} \sigma_{x}^{p}$. Measuring the product of these two observables simultaneously implies the discrimination of the four possible outcomes $\left(\sigma_{x}^{s} \sigma_{y}^{p}, \sigma_{y}^{s} \sigma_{x}^{p}\right)=$ $\{(+1,+1),(-1,-1),(+1,-1),(-1,+1)\}$, which is equivalent to a complete Bell-state discrimination [21, 22]. The two operators $\sigma_{x}^{s} \sigma_{y}^{p}$ and $\sigma_{y}^{s} \sigma_{x}^{p}$ have the four common Bell-like eigenstates

$$
\begin{aligned}
& \left|\varphi_{ \pm}\right\rangle=\frac{1}{\sqrt{2}}(|\downarrow\rangle \otimes|I\rangle \pm i|\uparrow\rangle \otimes|I I\rangle), \\
& \left|\phi_{ \pm}\right\rangle=\frac{1}{\sqrt{2}}(|\uparrow\rangle \otimes|I\rangle \pm i|\downarrow\rangle \otimes|I I\rangle),
\end{aligned}
$$

with the corresponding eigenvalue equations

$$
\begin{array}{rlrl}
\sigma_{x}^{s} \sigma_{y}^{p}\left|\varphi_{ \pm}\right\rangle & = \pm\left|\varphi_{ \pm}\right\rangle, & & \sigma_{y}^{s} \sigma_{x}^{p}\left|\varphi_{ \pm}\right\rangle=\mp\left|\varphi_{ \pm}\right\rangle, \\
\sigma_{x}^{s} \sigma_{y}^{p}\left|\phi_{ \pm}\right\rangle= \pm\left|\phi_{ \pm}\right\rangle, & & \sigma_{y}^{s} \sigma_{x}^{p}\left|\phi_{ \pm}\right\rangle= \pm\left|\phi_{ \pm}\right\rangle .
\end{array}
$$

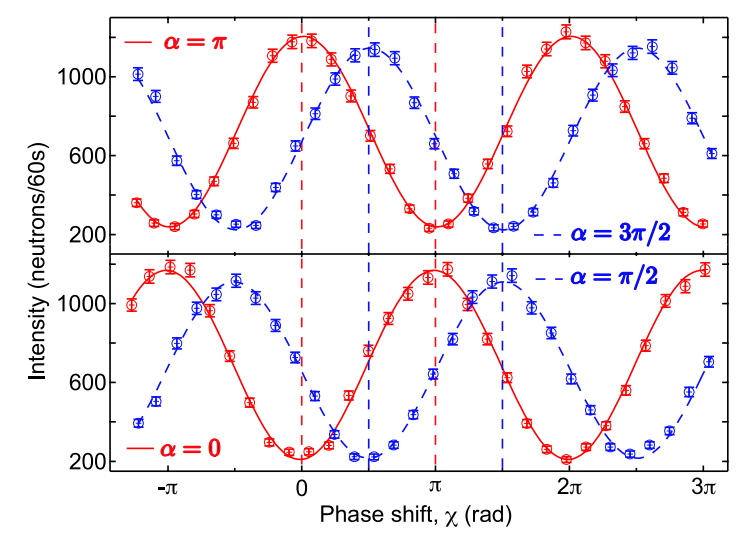

FIG. 2: Typical intensity modulations obtained by varying the phase $\chi$ for the path subspace. The contrast of the sinusoidal oscillations is above $67 \%$. The parameter $\alpha$ represents the direction of the spin analysis. In particular, the settings $\alpha=0, \pi\left[\alpha=\frac{\pi}{2}, \frac{3 \pi}{2}\right]$ were used for measuring $\sigma_{x}^{s}\left[\sigma_{y}^{s}\right]$. The expectation values, $E(0,0)\left[E\left(\frac{\pi}{2}, \frac{\pi}{2}\right)\right]$, are determined from the intensities on the dashed lines, $\chi=0, \pi\left[\chi=\frac{\pi}{2}, \frac{3 \pi}{2}\right]$.
It follows that the outcome -1 for the product measurement of $\sigma_{x}^{s} \sigma_{y}^{p}$ and $\sigma_{y}^{s} \sigma_{x}^{p}$ is obtained for $\left|\varphi_{ \pm}\right\rangle$, while the states $\left|\phi_{ \pm}\right\rangle$yield the result +1 . In practice, this Bell-state discrimination is accomplished by the following setup: $\mathrm{RF}_{\omega}^{I I}$ is tuned to flip the spin in path $I I$, i.e., transforming the state $|\Psi\rangle \rightarrow \frac{1}{\sqrt{2}}(|\downarrow\rangle \otimes|I\rangle-|\downarrow\rangle \otimes|I I\rangle)$. Note that $\mathrm{RF}_{\omega / 2}$ (used for compensating an energy difference between the two subbeams) is not needed for this measurement because the energy of the two sub-beams in the interferometer is the same after the spin flip in each path.

When the $\mathrm{DC}$ spin rotator in the $\mathrm{O}$ beam is adjusted to induce a $\pi$ flip, only $|\downarrow\rangle$-spin components reach the detector. Inducing a relative phase $\chi$ between the two beam paths in the interferometer allows then for projections to the state $|\varphi(\chi)\rangle=\frac{1}{\sqrt{2}}\left(|\downarrow\rangle \otimes|I\rangle+e^{i \chi}|\uparrow\rangle \otimes|I I\rangle\right)$. According to Eq. (6a), phase settings of $\chi= \pm \frac{\pi}{2}$ correspond to the measurement of $\left|\varphi_{ \pm}\right\rangle$. On the other hand, $|\uparrow\rangle$-spin analysis is achieved by switching the DC spin rotator off, where neutrons in the state $|\phi(\chi)\rangle=\frac{1}{\sqrt{2}}\left(|\uparrow\rangle \otimes|I\rangle+e^{i \chi}|\downarrow\rangle \otimes|I I\rangle\right)$ can be selected. Comparing with Eq. (6b), projections to $\left|\phi_{ \pm}\right\rangle$are obtained with the phase shifter settings $\chi= \pm \frac{\pi}{2}$. Typical intensity modulations for the two opposite settings of the spin analysis are shown in Fig. 3. Clear sinusoidal intensity oscillation is observed for analyzing $|\downarrow\rangle$ components, whereas the intensities with $|\uparrow\rangle$ spin analysis are marginal. The two relevant settings of the phase shifter, $\chi= \pm \frac{\pi}{2}$, are indicated by vertical dashed lines. The observed intensities reflect the quantum mechanical predictions for the measurement of the four Bell-like states given by the expectation values $\left\langle\Psi \mid \varphi_{ \pm}\right\rangle\left\langle\varphi_{ \pm} \mid \Psi\right\rangle=\frac{1}{2}$ and $\left\langle\Psi \mid \phi_{ \pm}\right\rangle\left\langle\phi_{ \pm} \mid \Psi\right\rangle=0$. Because of experimental imperfections, e.g., slightly less than $100 \%$ incident polarization and efficiencies of the spin flips, small contributions from the $\left|\phi_{ \pm}\right\rangle$components were found. Note that the setting of $\chi=\pi$ in the down-spin analysis yields the

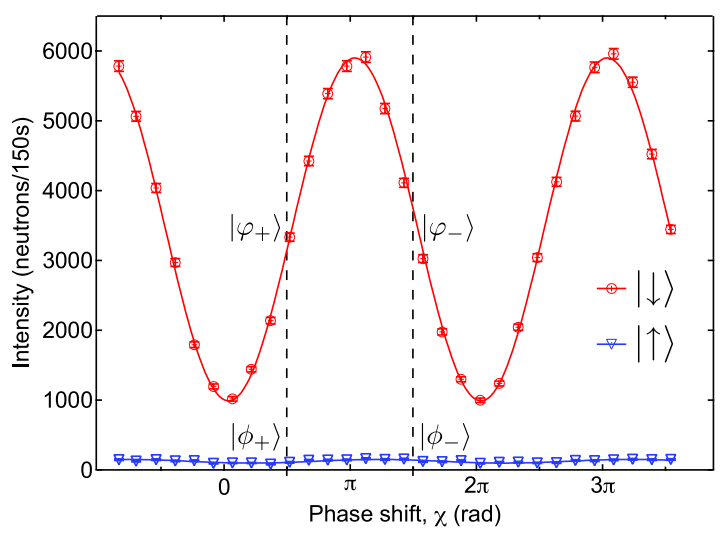

FIG. 3: Typical intensity modulations obtained by varying the phase $\chi$ in the path subspace. The two curves were recorded with opposite settings of the spin-analysis system selecting $|\downarrow\rangle$ - and $|\uparrow\rangle$ - components, respectively. Intensities on the dashed lines $\left(\chi=\frac{\pi}{2}, \frac{3 \pi}{2}\right)$ are used for the evaluation of the expectation value $\left\langle\sigma_{x}^{s} \sigma_{y}^{p} \cdot \sigma_{y}^{s} \sigma_{x}^{p}\right\rangle$. 
projection to $|\Psi\rangle=\frac{e^{i \pi / 4}}{\sqrt{2}}\left(\left|\varphi_{+}\right\rangle-i\left|\varphi_{-}\right\rangle\right)$. The intensity maximum located at this setting clearly proves the correct preparation of the state $|\Psi\rangle$. The expectation value $\left\langle\sigma_{x}^{s} \sigma_{y}^{p} \cdot \sigma_{y}^{s} \sigma_{x}^{p}\right\rangle$ is derived from the relation

$$
E^{\prime}=\frac{N^{\prime}\left(\phi_{+}\right)+N^{\prime}\left(\phi_{-}\right)-N^{\prime}\left(\varphi_{+}\right)-N^{\prime}\left(\varphi_{-}\right)}{N^{\prime}\left(\phi_{+}\right)+N^{\prime}\left(\phi_{-}\right)+N^{\prime}\left(\varphi_{+}\right)+N^{\prime}\left(\varphi_{-}\right)}
$$

where $N^{\prime}(\ldots)$ denotes the neutron count rate at the indicated projections. As before, least square fits were applied to deduce the count rates at the four projections. Because of thermal disturbances from the RF spin flippers in the interferometer, systematic shifts of up to $9^{\circ}$ of the measured oscillations were observed. Including all experimental errors in the error estimation, we determine the expectation value $\left\langle\sigma_{x}^{s} \sigma_{y}^{p} \cdot \sigma_{y}^{s} \sigma_{x}^{p}\right\rangle \equiv E^{\prime}=-0.93 \pm 0.003$.

With the three experimentally derived expectation values we can finally test inequality (44). We obtain $-\left\langle\sigma_{x}^{s} \cdot \sigma_{x}^{p}\right\rangle-\left\langle\sigma_{y}^{s} \cdot \sigma_{y}^{p}\right\rangle-\left\langle\sigma_{x}^{s} \sigma_{y}^{p} \cdot \sigma_{y}^{s} \sigma_{x}^{p}\right\rangle=2.291 \pm 0.008$, which is well above the upper limit of 1 given by the bound of NCHVTs. This result represents a violation of inequality (4) by 170 standard deviations. Moreover, the measured value is evidently closer to the quantum mechanical prediction of 3 than to the limit of NCHVTs.

As mentioned above, we also need to provide a prescription on how we could, at least in principle, test the experimental contexts of Eqs. (2C) and (2d). In case of testing Eq. (2C), the two possible outcomes of a measurement of $\sigma_{x}^{s} \sigma_{y}^{p}$ have to be discriminated with the same apparatus used to measure $\left\langle\sigma_{x}^{s} \sigma_{y}^{p} \cdot \sigma_{y}^{s} \sigma_{x}^{p}\right\rangle$. In order to perform the consecutive measurement of $\sigma_{x}^{s} \cdot \sigma_{y}^{p}$, the information of the comeasured observable $\sigma_{y}^{s} \sigma_{x}^{p}$ has to be erased. From the four output channels of the Bell-state discrimination apparatus, $\left|\varphi_{+}\right\rangle$and $\left|\phi_{+}\right\rangle\left(\left|\varphi_{-}\right\rangle\right.$and $\left.\left|\phi_{-}\right\rangle\right)$ correspond to the result $+1(-1)$ for the measurement of $\sigma_{x}^{s} \sigma_{y}^{p}$. It can be shown, that a coherent superposition of the $\left|\varphi_{+}\right\rangle$and $\left|\phi_{+}\right\rangle\left(\left|\varphi_{-}\right\rangle\right.$and $\left.\left|\phi_{-}\right\rangle\right)$components at a beam splitter with subsequent path and spin manipulation in form of unitary state rotations allows to preserve the information on the observable $\sigma_{x}^{s} \sigma_{y}^{p}$, while erasing any knowledge on the comeasured observable $\sigma_{y}^{s} \sigma_{x}^{p}$. The resulting sub-beams are then analyzed in path and spin degree of freedom with the same apparatus used for measuring $\left\langle\sigma_{x}^{s} \cdot \sigma_{x}^{p}\right\rangle$ and $\left\langle\sigma_{y}^{s} \cdot \sigma_{y}^{p}\right\rangle$. A similar experimental setup can be used for the measurement of $\left\langle\sigma_{y}^{s} \sigma_{x}^{p} \cdot \sigma_{y}^{s} \cdot \sigma_{x}^{p}\right\rangle$. With these configurations, the two missing experimental contexts of Eqs. (2c) and (2d) can be tested.

Although independent manipulation of energy and spin degrees of freedom in neutron interferometry were reported [16, 17], these two Hilbert subspaces are always coupled in the experiment performed here and only spinpath entangled states need to be considered.

In summary, we entangled the spin and the path degrees of freedom of single neutrons in neutron interferometry for testing an inequality based on the Peres-Mermin proof of the Kochen-Specker theorem. The three expectation values required for the proof were obtained in sequential measurements. In particular, one of the expectation values was derived from a Bell-state discrimination method. The observed value of $2.291 \pm 0.008 \not \leq 1$ clearly confirms the conflict with NCHVTs.

We appreciate helpful discussions with R. A. Bertlmann and K. Durstberger-Rennhofer (Vienna). This work was supported by the Japanese Science and Technology Agency, the Austrian Fonds zur Förderung der Wissenschaftlichen Forschung, the MCI Project No. FIS2008-05596, and the Junta de Andalucía Excellence Project No. P06-FQM-02243.
[1] J. S. Bell, Physics (Long Island City, N. Y.) 1, 195 (1964).

[2] A. Aspect, P. Grangier, and G. Roger, Phys. Rev. Lett. 49, 91 (1982).

[3] G. Weihs, T. Jennewein, C. Simon, H. Weinfurter, and A. Zeilinger, Phys. Rev. Lett. 81, 5039 (1998).

[4] M. A. Rowe, D. Kielpinski, V. Meyer, C. A. Sackett, W. M. Itano, C. Monroe, and D. J. Wineland, Nature (London) 409, 791 (2001).

[5] T. Scheidl, R. Ursin, J. Kofler, S. Ramelow, X.-S. Ma, T. Herbst, L. Ratschbacher, A. Fedrizzi, N. Langford, T. Jennewein, and A. Zeilinger, arXiv:0811.3129 [Nat. Phys. (to be published)].

[6] Y. Hasegawa, R. Loidl, G. Badurek, M. Baron, and H. Rauch, Nature (London) 425, 45 (2003).

[7] S. Kochen and E. Specker, J. Math. Mech. 17, 59 (1967).

[8] A. Peres, Phys. Lett. A 51, 107 (1990).

[9] N. D. Mermin, Phys. Rev. Lett. 65, 3373 (1990).

[10] N. D. Mermin, Rev. Mod. Phys. 65, 803 (1993).

[11] A. Cabello, S. Filipp, H. Rauch, and Y. Hasegawa, Phys. Rev. Lett. 100, 130404 (2008).

[12] A. Cabello, Phys. Rev. Lett. 101, 210401 (2008).

[13] H. Rauch, W. Treimer, and U. Bonse, Phys. Lett. A 47,
369 (1974)

[14] H. Rauch and S. A. Werner, Neutron Interferometry (Clarendon, Oxford, 2000).

[15] Y. Hasegawa, R. Loidl, G. Badurek, S. Filipp, J. Klepp, and H. Rauch, Phys. Rev. A 76, 052108 (2007).

[16] S. Sponar, J. Klepp, R. Loidl, S. Filipp, G. Badurek, Y. Hasegawa, and H. Rauch, Phys. Rev. A 78, 061604(R) (2008).

[17] Y. Hasegawa, R. Loidl, G. Badurek, S. Sponar, and H. Rauch (to be published).

[18] J. Klepp, S. Sponar, S. Filipp, M. Lettner, G. Badurek, and Y. Hasegawa, Phys. Rev. Lett. 101, 150404 (2008).

[19] S. Filipp, J. Klepp, Y. Hasegawa, C. Plonka-Spehr, U. Schmidt, P. Geltenbort, and H. Rauch, Phys. Rev. Lett. 102, 030404 (2009).

[20] Y. Hasegawa, R. Loidl, G. Badurek, M. Baron, and H. Rauch, Phys. Rev. Lett. 97, 230401 (2006).

[21] T. Yang, Q. Zhang, J. Zhang, J. Yin, Z. Zhao, M. Żukowski, Z.-B. Chen, and J.-W. Pan, Phys. Rev. Lett. 95, 240406 (2005).

[22] M. Barbieri, G. Vallone, P. Mataloni, and F. De Martini, Phys. Rev. A 75, 042317 (2007). 